\title{
Obesity and binge alcohol intake are deadly combina- tion to induce steatohepatitis: A model of high-fat diet and binge ethanol intake
}

\author{
Seonghwan Hwang*, Tianyi Ren*, and Bin Gao \\ Laboratory of Liver Diseases, National Institute on Alcohol Abuse and Alcoholism, National Institutes of Health, Bethesda, MD, USA
}

Obesity and binge drinking often coexist and work synergistically to promote steatohepatitis; however, the underlying mechanisms remain obscure. In this mini-review, we briefly summarize clinical evidence of the synergistical effect of obesity and heavy drinking on steatohepatitis and discuss the underlying mechanisms obtained from the study of several mouse models. High-fat diet (HFD) feeding and binge ethanol synergistically induced steatohepatitis and fibrosis in mice with significant intrahepatic neutrophil infiltration; such HFD-plus-ethanol treatment markedly up-regulated the hepatic expression of many chemokines with the highest fold (approximately 30-fold) induction of chemokine (C-X-C motif) ligand $1(C x / 1)$, which contributes to hepatic neutrophil infiltration and liver injury. Furthermore, HFD feeding activated peroxisome proliferator-activated receptor gamma that subsequently inhibited CXCL1 upregulation in hepatocytes, thereby forming a negative feedback loop to prevent neutrophil overaction; whereas binge ethanol blocked this loop and then exacerbated CXCL1 elevation, neutrophil infiltration, and liver injury. Interestingly, inflamed mouse hepatocytes attracted neutrophils less effectively than inflamed human hepatocytes due to the lower induction of CXCL1 and the lack of the interleukin (IL)-8 gene in the mouse genome, which may be one of the reasons for difficulty in development of mouse models of alcoholic steatohepatitis and nonalcoholic steatohepatitis (NASH). Hepatic overexpression of Cxcl1 and/or IL-8 promoted steatosis-to-NASH progression in HFD-fed mice by inducing neutrophil infiltration, oxidative stress, hepatocyte death, fibrosis, and p38 mitogen-activated protein kinase activation. Collectively, obesity and binge drinking synergistically promote steatohepatitis via the induction of CXCL1 and subsequent hepatic neutrophil infiltration. (Clin Mol Hepatol 2020;26:586-594)

Keywords: Chemokine CXCL1; Neutrophils; NASH; p38 mitogen-activated protein kinase

\footnotetext{
Abbreviations:

CVD, cardiovascular disease; CXCL1, C-X-C motif chemokine ligand 1; ER endoplasmic reticulum; FFA, free fatty acid; FSP27, fat-specific protein 27; HFD, high-fat diet; HSCs, hepatic stellate cells; ICAM-1, intercellular adhesion molecule 1; IL, interleukin; JNK, c-Jun N-terminal kinase; MAPK, mitogen-activated protein kinase; NAFLD, nonalcoholic fatty liver disease; NASH, nonalcoholic steatohepatitis; ASH, alcoholic steatohepatitis; NF-kB, nuclear factor kappalight-chain-enhancer of activated B cells; PPAR-g, peroxisome proliferatoractivated receptor gamma; ROS, reactive oxygen species; ASK1, apoptosis signalregulating kinase 1

*SH and TR contribute equally to write this review article.
}

Corresponding author : Bin Gao

Laboratory of Liver Diseases, National Institute on Alcohol Abuse and Alcoholism, National Institutes of Health, 5625 Fishers Lane, Bethesda, MD 20892, USA

Tel: +1-301-443-3998, Fax: +1-301-480-0257

E-mail:bgao@mail.nih.gov

https://orcid.org/0000-0002-0505-2972 


\section{INTRODUCTION}

Although obesity and heavy drinking, which often coexist in patients, have been shown to promote the development of liver injury, steatohepatitis, and fibrosis, ${ }^{1,2}$ the clinical data on light-tomoderate alcohol drinking on nonalcoholic fatty liver disease (NAFLD) have been controversial. ${ }^{3,4}$ The data from experimental models on the effect of chronic alcohol feeding on high-fat diet (HFD)-induced NAFLD are limited because it is difficult to manage chronic alcohol and HFD feeding together due to marked reduction of ad libitum HFD intake by chronic ethanol feeding in mice. Xu et al. ${ }^{5}$ performed intragastric force-feeding of HFD-plus-ethanol in mice and found HFD and ethanol synergistically induce nitrosative, endoplasmic reticulum (ER), and mitochondrial stress and an upregulation of hepatic toll-like receptor 4 , thereby contributing to steatohepatitis. Recently, we developed a model of HFD-plus-binge ethanol challenge, ${ }^{6,7}$ which mimics binge drinking and obesity in humans. Our data revealed that binge alcohol intake and HFD synergistically induce steatohepatitis and fibrosis. ${ }^{6,7}$ In the current review, we briefly describe clinical data on the effects of obesity and alcohol drinking on steatohepatitis and deliberate the underlying mechanisms obtained from the study of several mouse models.

\section{CLINICAL DATA ON THE EFFECTS OF OBESITY AND ALCOHOL DRINKING ON NAFLD}

Recent increases in the incidence of obesity and metabolic syndrome have highlighted the potential synergism between obesity and heavy drinking to cause liver injury and resultant inflammation. ${ }^{1,2}$ Several clinical studies have documented the additive effects of obesity and heavy/moderate alcohol drinking on steatohepatitis and liver-related death. ${ }^{8-13}$ For example, in a cohort study of 233 alcoholic hepatitis patients, obesity was found to be associated with a greater than two-fold increase in short-term mortality. ${ }^{2}$ However, the clinical data on the effects of light to moderate alcohol drinking on NAFLD have been controversial. Some early observational studies suggest low to moderate alcohol use does not increase or even reduces the risk of fatty liver ${ }_{i}^{14-16}$ but this conclusion was later questioned by meta analyses. ${ }^{4,17}$ The crosssectional profile of these studies make it impossible to establish temporal relationships between alcohol consumption and NAFLD. A Korean cohort study followed 190,048 adults without NAFLD at baseline for 15.7 years and documented a decreased risk of hepatic steatosis in moderate drinkers. ${ }^{18}$ However, in the subgroup of participants who developed more severe NAFLD over time, low levels of alcohol consumption were associated with the risk of liver fibrosis, especially for nonobese individuals. ${ }^{18}$ Besides the impact on hepatic steatosis incidence, the impact of low to moder-

Table 1. Recent clinical studies on liver disease caused by combination of ethanol and obesity

\begin{tabular}{|c|c|c|}
\hline Year & Conclusion & Reference \\
\hline 2008 & Modest wine consumption is associated with reduced prevalence of suspected NAFLD. & 16 \\
\hline 2010 & Obesity and alcohol synergistically contribute to the risk of liver-related death. & 58 \\
\hline 2011 & Modest alcohol consumption does not increase the risk of fatty liver or liver fibrosis. & 14 \\
\hline 2014 & Light to moderate alcohol consumption is protective against NAFLD development over time. & 15 \\
\hline 2017 & $\begin{array}{l}\text { No association was observed between alcohol use and the presence of cardiovascular disease risk factors in NAFLD } \\
\text { individuals. }\end{array}$ & 22 \\
\hline 2018 & Low to moderate alcohol use increases liver related death in NASH. & 20 \\
\hline 2018 & In NAFLD population, $0.5-1.5$ drinks per day reduce overall mortality, whereas $\geq 1.5$ drinks per day become harmful. & 21 \\
\hline 2018 & Moderate alcohol use was associated with less improvement in steatosis, as well as lower odds of NASH resolution. & 19 \\
\hline 2019 & In patients with severe alcoholic liver disease, obesity is a risk factor for short-term morbidity and mortality. & 2 \\
\hline 2019 & Moderate drinking was associated with worsening of noninvasive markers of fibrosis in NAFLD. & 10 \\
\hline 2020 & $\begin{array}{l}\text { Modest alcohol consumption is associated with a decreased risk of hepatic steatosis, while an increased risk of } \\
\text { hepatic steatosis plus fibrosis (especially in nonobese individuals). }\end{array}$ & 18 \\
\hline 2020 & $\begin{array}{l}\text { Even low alcohol intake in fatty liver is associated with increased risks of advanced liver disease and cancer, but a } \\
\text { lower risk of cardiovascular disease incidence. }\end{array}$ & 8 \\
\hline
\end{tabular}

NAFLD, nonalcoholic fatty liver disease; NASH, nonalcoholic steatohepatitis. 
Table 2. Recent experimental studies on liver disease caused by combination of ethanol and obesity

\begin{tabular}{|c|c|c|}
\hline Year & Conclusion & Reference \\
\hline 2011 & $\begin{array}{l}\text { Intragastric feeding of HFD and ethanol synergistically cause steatohepatitis through nitrosative stress mediated by } \\
\text { M1 macrophage activation, adiponectin resistance, and enhanced ER and mitochondrial stress in mice. }\end{array}$ & 5 \\
\hline 2013 & $\begin{array}{l}\text { Ethanol feeding potentiates hepatic lipid oxidation in ob/ob mice through dysregulation of sirtuin 1-AMP-activated } \\
\text { protein kinase signaling. }\end{array}$ & 59 \\
\hline 2014 & Binge alcohol consumption exacerbates oxidative stress and promotes steatosis-to-NASH development in rats. & 60 \\
\hline 2014 & Alcohol and fructose synergistically enhance dyslipidemia and insulin resistance-associated liver damage in rats. & 61 \\
\hline 2015 & $\begin{array}{l}\text { HFD and ethanol binge synergistically induce acute steatohepatitis by inducing CXCL1-mediated hepatic neutrophil } \\
\text { infiltration in mice: a mouse model of acute liver induced by obesity and binge drinking. }\end{array}$ & 6 \\
\hline 2015 & Multiple ethanol binges exacerbate HFD-induced liver steatosis, inflammation, and fibrosis in mice. & 62 \\
\hline 2016 & $\begin{array}{l}\text { Fructose potentiates chronic alcohol-induced liver injury through activation of toll-like receptor } 4 \text { signaling pathway } \\
\text { and M1 macrophage polarization in mice. }\end{array}$ & 63 \\
\hline 2017 & Chronic-plus-binge ethanol-induced liver injury is potentiated by linoleic acid and its metabolites in mice. & 64 \\
\hline 2018 & Ethanol and HFD synergistically induce hepatic fibrosis via neutrophil-hepatic stellate cell interactions in mice. & 7 \\
\hline
\end{tabular}

HFD, high-fat diet; ER, endoplasmic reticulum; NASH, nonalcoholic steatohepatitis; CXCL1, C-X-C motif chemokine ligand 1.

ate drinking on NAFLD development and relevant clinical outcomes has received fierce debate as well. Targeting biopsyconfirmed NAFLD patients, moderate drinking was reported to bring about lower odds for histological improvement and nonalcoholic steatohepatitis (NASH) resolution. ${ }^{19}$ In a large-scale Korean cohort of young and middle-aged NAFLD individuals, nonheavy alcohol use was associated with noninvasive fibrosis indexes, indicating the perniciousness of even modest drinking. ${ }^{10}$ Longitudinal observation on more severe NAFLD cases with fibrosis or cirrhosis further demonstrated the facilitation of low to moderate drinking to liver decompensation and liver-related death. ${ }^{20}$ A recent study on Finland population also showed a correlation between alcohol intake (even at low levels) and the risk for advanced liver diseases on the basis of fatty liver. But as a whole, low to moderate drinking was associated with reduced mortality and cardiovascular disease (CVD) risk in NAFLD patients. ${ }^{8}$ Similarly, a prospective study based on 4,568 USA subjects with NAFLD (defined by the hepatic steatosis index) noted significant protective effects of $0.5-1.5$ drinks per day against allcause mortality, whereas $\geq 1.5$ drinks per day led to an increase in mortality. ${ }^{21}$ In contrast, a longitudinal cohort study following 570 USA individuals with NAFLD found prospectively assessed alcohol use was not associated with significant differences in CVD risk. ${ }^{22}$ These results were further challenged by the Global Burden of Disease study, which indicated even light drinking increases CVD risk and mortality. ${ }^{23}$ Given such controversy and disagreement regarding the effects of alcohol on NAFLD, further rigor clinical and mechanistic studies are in urgent need. Table 1 summarized re- cent clinical studies on liver disease caused by combination of alcohol drinking and obesity.

\section{HFD-PLUS-ETHANOL BINGE MODEL INDUCES SEVERE STEATOHEPATITIS}

Investigators have recently performed experimental studies to better understand the synergistic role of ethanol and obesity and the associated mechanisms for the potentiation of liver disease by ethanol and obesity as summarized in Table 2. Because chronic alcohol feeding markedly reduces ad libitum HFD intake in mice, it is difficult to manage chronic alcohol and HFD feeding together unless performing intragastric force-feeding. ${ }^{5}$ Recently, we developed a model to study the interaction of obesity and binge drinking by introducing binge alcohol intake in HFD-fed mice, and our data suggest that binge ethanol intake and HFD synergistically induced steatohepatitis as shown by a marked increase in hepatic neutrophil infiltration and inflammatory markers as well as liver injury (Fig. 1). ${ }^{6}$

Hepatic neutrophil infiltration is a critical pathologic feature during liver injury, usually serving as a hallmark of both obesity and alcohol-related liver diseases. ${ }^{24} \mathrm{C}-\mathrm{X}$-C motif chemokine ligand 1 (CXCL1) belongs to the CXC chemokine family and is the major chemokine mediating neutrophil recruitment through CXCR2. ${ }^{25}$ In patients with alcoholic hepatitis, both hepatic and serum levels of CXCL1 were reported markedly elevated. Meanwhile, hepatic CXCL1 mRNA levels correlated positively with hepatic neutrophil 
Seonghwan Hwang, et al. Obesity and binge drinking induce steatohepatitis

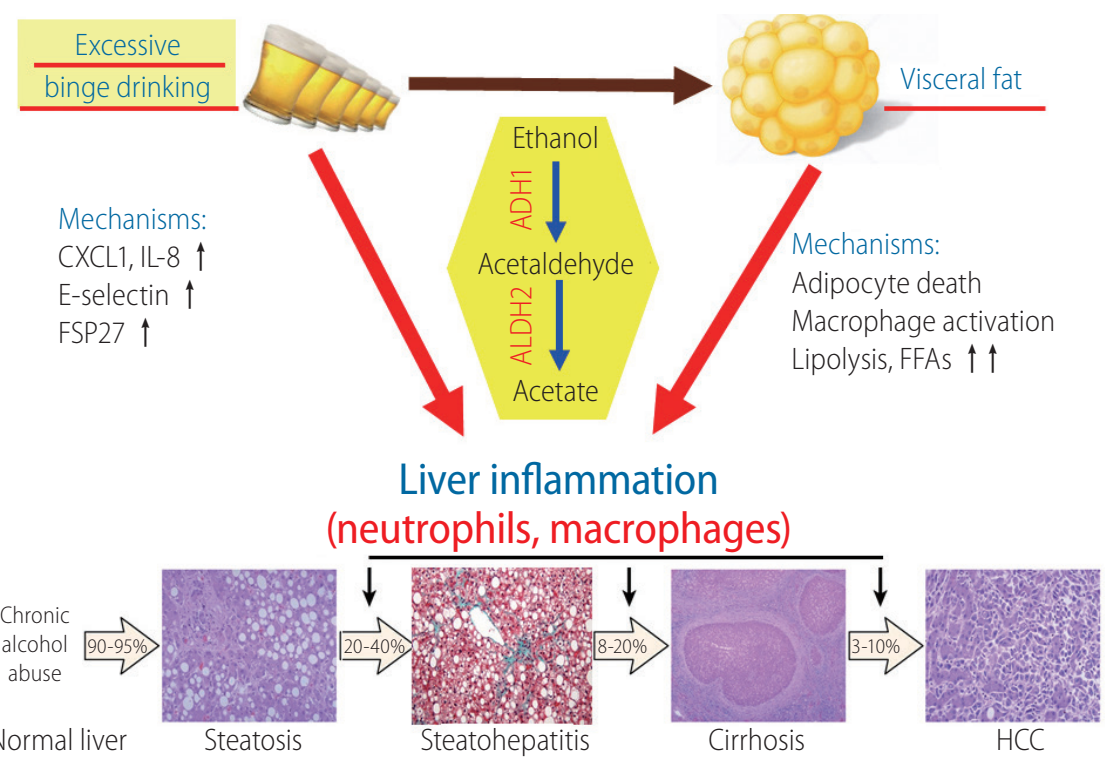

Figure 1. Binge drinking and fat synergistically promote ALD. Ingested ethanol is metabolized to acetaldehyde and acetate by the sequential action of $\mathrm{ADH} 1$ and $\mathrm{ALDH} 2$, respectively. Excessive binge drinking induces neutrophil-recruiting chemokines such as CXCL1 and IL-8 and endothelial cell adhesion molecule E-selectin, thereby allowing for the enhanced infiltration of neutrophils in the liver. Binge drinking also results in mitochondrial DNA damage and induction of fat-accumulating protein FSP27 in the liver. In cooperation with these factors that enhance liver injury and inflammation, alcohol-induced dysregulation of visceral fat potentiates the liver inflammation through mechanisms involving adipocyte death, macrophage activation, lipolysis, and FFA release. IL-8, interleukin 8; FSP27, fat-specific protein 27; ADH1, alcohol dehydrogenase 1; ALDH2, aldehyde dehydrogenase 2; FFA, free fatty acid; HCC, hepatocellular carcinoma; ALD, alcohol-related liver disease; CXCL1, C-X-C motif chemokine ligand 1.

infiltration in the same group of patients. ${ }^{26}$ More remarkably, the CXCL1 rs4074 A allele in human has been associated with elevated serum CXCL1 levels and considered as a genetic risk factor for alcoholic cirrhosis. ${ }^{27} \mathrm{CXCl}$ was one of the most highly-induced genes in the liver of mice upon challenge with the combination of 3-month HFD-plus-binge ethanol (30-fold in the liver and 5-fold in epididymal adipose tissue). ${ }^{6}$ In the liver, elevation of $C x \mathrm{Cl} 1 \mathrm{ex}$ pression was the most significant in hepatocytes, and to a lesser extent in hepatic stellate cells (HSCs) and liver sinusoidal endothelial cells. ${ }^{6}$ The induction of CXCL1 by HFD-plus-ethanol binge is believed to be associated with the increased levels of free fatty acid (FFA) in the liver, which upregulate $C X C / 1$ in hepatocytes in a manner depending on extracellular signal-regulated kinase $1 / 2$, c-Jun N-terminal kinase (JNK), and nuclear factor kappa-lightchain-enhancer of activated B cells (NF-KB) signaling. Blockade of CXCL1 with a neutralizing antibody or disrupting the $C X C / 1$ gene ameliorated hepatic neutrophil infiltration and injury after HFDplus-ethanol binge, whereas overexpression of $C x c / 1$ exacerbated steatohepatitis in 3-motnh HFD-fed mice.

Peroxisome proliferator-activated receptor gamma (PPAR- $\gamma$ ) expression in the liver is very low under physiological conditions, but markedly upregulated in fatty liver animal models and NAFLD pa- tients. ${ }^{28}$ Disruption of the Pparg gene in hepatocytes ameliorates steatosis, whereas its overexpression promotes the development of fatty liver through the activation of various lipogenic genes, including fat-specific protein 27 (FSP27), ${ }^{29}$ and the resultant lipogenesis. ${ }^{30}$ The steatohepatitis model featuring the combination of HFD-plus-ethanol binge identified the dichotomous role of PPAR- $\gamma$ underlying the synergistic effect of HFD and ethanol binge on steatohepatitis: 1) HFD feeding activates PPAR- $\gamma$, and FFA upregulates hepatic CXCL1 expression; 2) PPAR- $y$ inhibits NF-KB-mediated $\mathrm{Cx} / 1$ expression and subsequently prevents hepatic neutrophil infiltration; and 3) ethanol binge blocks this negative feedback loop and increases the NF-KB/CXCL1 pathway, which consequently exacerbates neutrophil infiltration and liver injury. In addition, this HFD-plus-binge ethanol model has been recently used to demonstrate that $\gamma \delta \mathrm{T}$ cells play a central role in steatohepatitis by mitigating $T$ cell expansion and modulating their inflammatory program. ${ }^{31,32}$ This model was also recently used to test some therapies for alcohol-induced liver toxicity. ${ }^{33}$

Although this HFD-plus-ethanol binge model does not represent chronic alcoholic steatohepatitis (ASH), it can trigger acute liver injury and mimic hepatic neutrophil infiltration as typically shown in ASH patients. ${ }^{34,35}$ Its obvious clinical implication is that obese 


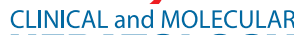

\section{HEPATOLOGY}

Volume_26 Number_4 October 2020

individuals should avoid binge drinking of large amount of alcohol, as this would likely cause significant acute liver damage. Furthermore, the rapidity of the development of neutrophil-mediated steatohepatitis, which would make it uniquely suitable for future mechanistic studies.

\section{HFD-PLUS-ETHANOL BINGE PROMOTES LIVER FIBROSIS}

During steatohepatitis, persistent inflammation dysregulates liver homeostasis and activates the repair processes. ${ }^{36}$ Activated HSCs secrete extracellular matrix to support the injured region and construct the regeneration scaffold for subsequent hepatocyte proliferation (i.e., liver fibrosis formation), along with the clearance of necrotic tissues by infiltrating leukocytes. ${ }^{37}$ Fibrosis has been postulated to be promoted by neutrophils that induce hepatocellular damage and HSC activation via the production of reactive oxygen species (ROS). ${ }^{36} \mathrm{HFD}$-plus-ethanol binge that features the hepatic infiltration of neutrophils caused a marked upregulation of an array of genes related to HSC activation and fibrogenesis compared with HFD feeding only. ${ }^{7}$ Blockade of CXCL1 with a neutralizing antibody or genetic disruption of the $\mathrm{CxC} / 1$ gene markedly attenuated liver fibrosis in the HFD-plus-ethanol binge model, and disruption of the intercellular adhesion molecule 1 (ICAM-1) gene that encodes ICAM-1 protein, a key adhesion molecule for neutrophil recruitment, attenuated neutrophil infil- tration and liver fibrosis caused by HFD-plus- binge ethanol, indicating the significant involvement of infiltrated neutrophils in mediating fibrogenesis. ${ }^{7}$ Mechanistically, ROS production mediated by $p 47^{\text {phox }}$, an important component of nicotinamide adenine dinucleotide phosphate oxidase 2 complex that mediates neutrophil oxidative burst, ${ }^{38}$ potentiates HSC activation, and activated HSCS act against neutrophils death via the strong production of granulocyte-macrophage colony-stimulating factor and interleukin (IL)15, which are growth factors that play crucial roles in sustaining the survival of neutrophils. ${ }^{39,40}$ This result indicates that binge drinking potentiates fibrogenesis in mice fed an $\mathrm{HFD}_{1}{ }_{1}^{11}$ expanding on the synergism between obesity and binge drinking in exacerbation of liver injury and neutrophil infiltration.

\section{ADIPOSE TISSUE DYSFUNCTION CONTRIBUTES TO ALCOHOL-INDUCED LIVER INJURY}

In addition to the synergism between hepatic fat and alcohol toxicity in the liver, the crosstalk between the adipose tissue and the liver has been highlighted as the cause for worsening alcoholinduced liver injury in obese individuals.' It has been recently reported that alcohol consumption impairs the function of adipose tissue through induction of adipocyte apoptosis and subsequent inflammation as well as lipolysis of adipocytes, reduction in insulin-dependent glucose uptake, and dysregulation of adipokines and cytokines (Fig. 2). ${ }^{42,43}$ A study utilizing a mouse model of in-

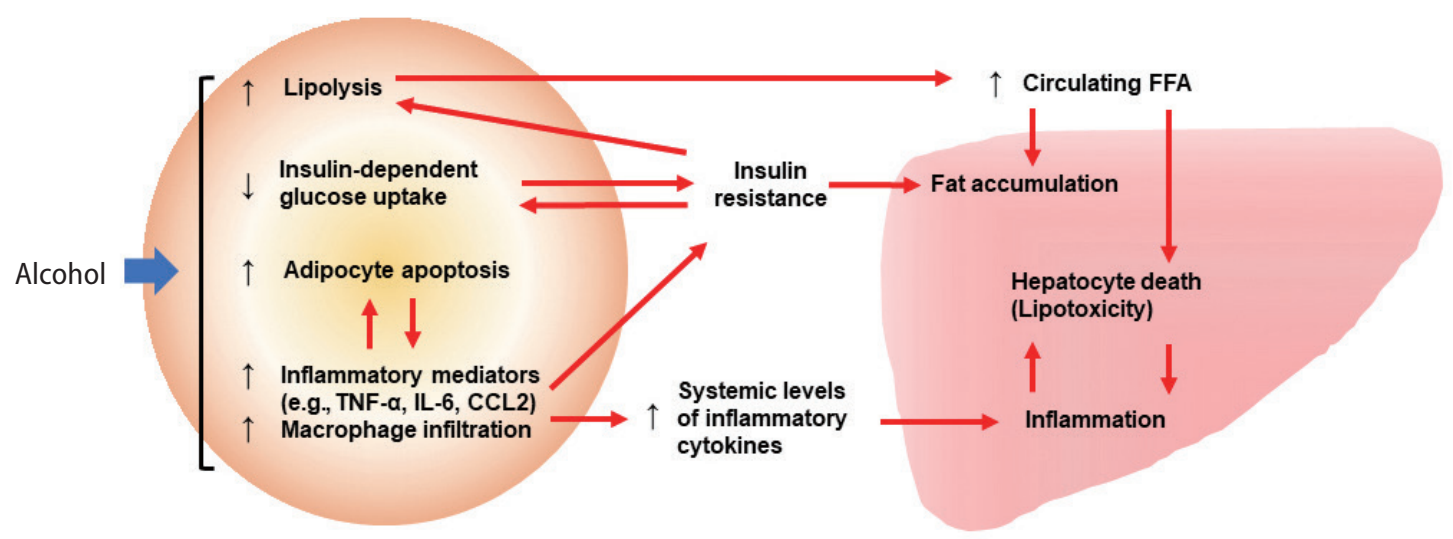

White adipose tissue

Liver

Figure 2. Role of white adipose tissue in the pathogenesis of ALD. Excessive alcohol intake induces white adipocyte death and white adipose tissue inflammation, resulting in elevation of epinephrine and norepinephrine, and subsequent lipolysis. Alcohol and dysfunctional white adipocytes can trigger insulin resistance, which also promotes lipolysis. White adipose tissue lipolysis causes elevation of circulating FFA levels, thereby inducing hepatic FFA influx, lipotoxicity, steatosis, hepatocyte death, and liver inflammation. Modified from Hwang and Gao. ${ }^{42}$ TNF-a, tumor necrosis factor-alpha; IL-6, interleukin-6; CCL2, chemokine (C-C) motif ligand 2; FFA, free fatty acid; ALD, alcohol-related liver disease. 
Seonghwan Hwang, et al. Obesity and binge drinking induce steatohepatitis

ducible adipocyte death demonstrated that acute adipocyte death results in liver injury and inflammation via lipolysis and the activation of chemokine (C-C motif) receptor 2-positive macrophages, ${ }^{44}$ supporting the importance of adipocyte dysfunction in alcohol-induced liver injury.

\section{OVEREXPRESSION OF CXCL1 PROMOTES STE- ATOSIS-TO-NASH PROGRESSION}

Obesity and insulin resistance are major risk factors for NAFLD, which ranges from steatosis to NASH, cirrhosis, and hepatocellular carcinoma. ${ }^{45,46}$ Steatosis features excessive accumulation of fat in the liver without signs of severe inflammation or liver injury. ${ }^{47}$ Approximately $25 \%$ of patients with steatosis further progress to NASH, which is defined by the presence of liver injury (hepatocyte ballooning), inflammation, and fibrosis in addition to hepatic fat deposition. ${ }^{47,48}$ Diet-induced obesity model such as 3-month HFD feeding in mice readily results in the development of steatosis with gene expression profile and histological features similar to those of human steatosis; however, mice are relatively resistant to NASH development under this condition.

A key feature of human NASH is the marked infiltration of neutrophils in the liver and enhanced hepatic expression of CXCL1 and IL-8, major chemokines for neutrophil chemotaxis, ${ }^{49}$ which are not commonly observed in patients with fatty liver or mice subjected to HFD-induced fatty liver model. However, it has been elusive whether neutrophils play a critical role in steatosis-toNASH progression, and if so, how infiltrated neutrophils contribute to the development of NASH.

Adenovirus-mediated overexpression of $\mathrm{Cx} / 1$ significantly elevated neutrophil infiltration in the liver of mice fed an HFD for 3 months. ${ }^{50}$ Increased neutrophil population promoted ROS production which led to an activation of apoptosis signal-regulating kinase 1 (ASK1) and p38 mitogen-activated protein kinase (MAPK), thereby activating the signaling pathways leading to apoptosis- and ER stress-induced hepatocyte death. ${ }^{51}$ DNA damage, oxidative stress, and other intrinsic apoptotic stimuli incurred by infiltrated neutrophils activate the mitochondrial apoptotic pathway of hepatocytes. ROS production can also disturb the protein folding process within the ER lumen by changing the redox status, which further cause ER stress. ${ }^{52,53}$ It has been also demonstrated that infiltrated neutrophils cause hepatocyte death through $p 47^{\text {phox }}$-dependent oxidative burst and subsequent activation of ROS-sensitive stress kinases such as p38 MAPK and JNK, ${ }^{50}$ which in turn facilitates inflammatory and fibrogenic processes and exacerbates steatosis-to-NASH progression. Microarray analysis revealed that HFD/CXCL1-induced NASH possessed expression profiles of inflammatory and fibrogenic genes, which are similar to those of NASH patients, supporting the notion that $\mathrm{CxC} / 1$ overexpression results in steatosis-to-NASH progression in HFD-fed mice. $^{50}$

Experimental models widely used to study NASH are not developed based on the molecular mechanisms that promote steatosisto-NASH progression, but mostly developed by modulating the components of diet as seen in high-fat high-cholesterol diet, methionine choline-deficient diet, and HFD-plus-chronic $\mathrm{CCl}_{4}$ treatment. ${ }^{54,55}$ Hepatic neutrophil infiltration is commonly observed in steatohepatitis with different etiologies including alcohol consumption and metabolic dysregulation, and thus experimental model exploiting this hallmark of steatohepatitis will better recapitulate the spectrum of NAFLD progression in human and serve as a useful tool to investigate the pathogenic mechanisms of NASH as well as a novel therapeutic target.

\section{CONCLUSION}

Emerging data from experimental models clearly suggest that binge ethanol intake and obesity synergistically induce steatohepatitis in mice via the induction of hepatic CXCL1 and neutrophil infiltration. So far, the effects of binge drinking and obesity on steatohepatitis in humans have not been carefully studied. Interestingly, we recently demonstrated that inflamed human hepatocytes attracted neutrophils more effectively than inflamed mouse hepatocytes due to the greater induction of CXCL1 and IL-8 in human hepatocytes (lack of the IL- 8 gene in the mouse genome). ${ }^{51}$ In addition, the number of circulating neutrophils in humans $\left(\sim 4 \times 10^{9} / \mathrm{L}\right)$ is much higher than in mice $\left(\sim 1 \times 10^{9} / \mathrm{L}\right) .{ }^{56}$ Finally, ethanol metabolism in humans is five times slower than that in rodents. ${ }^{57}$ Based on these factors, it is plausible to speculate that the synergistic effect of binge drinking and obesity on steatohepatitis in humans may be more significant than that in mice. Thus, obese individuals should avoid binge drinking large amount of alcohol as this would likely cause significant acute liver damage.

\section{Authors' contributions}

SH and TR wrote the paper, BG supervised the whole project and edited the paper. 


\section{Acknowledgements}

The work from Dr. Bin Gao's lab described in the current paper was supported by the intramural program of NIAAA, NIH (B.G.).

\section{Conflicts of Interest}

The authors have no conflicts to disclose.

\section{REFERENCES}

1. Parker R, Kim SJ, Gao B. Alcohol, adipose tissue and liver disease: mechanistic links and clinical considerations. Nat Rev Gastroenterol Hepatol 2018;15:50-59.

2. Parker R, Kim SJ, Im GY, Nahas J, Dhesi B, Vergis N, et al. Obesity in acute alcoholic hepatitis increases morbidity and mortality. EBioMedicine 2019;45:511-518.

3. Kwon I, Jun DW, Moon JH. Effects of moderate alcohol drinking in patients with nonalcoholic fatty liver disease. Gut Liver 2019;13:308-314.

4. Ajmera VH, Terrault NA, Harrison SA. Is moderate alcohol use in nonalcoholic fatty liver disease good or bad? A critical review. Hepatology 2017;65:2090-2099.

5. Xu J, Lai KKY, Verlinsky A, Lugea A, French SW, Cooper MP, et al. Synergistic steatohepatitis by moderate obesity and alcohol in mice despite increased adiponectin and p-AMPK. J Hepatol 2011;55:673682.

6. Chang B, Xu MJ, Zhou Z, Cai Y, Li M, Wang W, et al. Short- or longterm high-fat diet feeding plus acute ethanol binge synergistically induce acute liver injury in mice: an important role for CXCL1. Hepatology 2015;62:1070-1085.

7. Zhou Z, Xu MJ, Cai Y, Wang W, Jiang JX, Varga ZV, et al. Neutrophilhepatic stellate cell interactions promote fibrosis in experimental steatohepatitis. Cell Mol Gastroenterol Hepatol 2018;5:399-413.

8. Åberg F, Puukka P, Salomaa V, Männistö S, Lundqvist A, Valsta L, et al. Risks of light and moderate alcohol use in fatty liver disease: follow-up of population cohorts. Hepatology 2020;71:835-848.

9. Alatalo PI, Koivisto HM, Hietala JP, Puukka KS, Bloigu R, Niemelä OJ. Effect of moderate alcohol consumption on liver enzymes increases with increasing body mass index. Am J Clin Nutr 2008:88:10971103.

10. Chang Y, Cho YK, Kim Y, Sung E, Ahn J, Jung HS, et al. Nonheavy drinking and worsening of noninvasive fibrosis markers in nonalcoholic fatty liver disease: a cohort study. Hepatology 2019;69:64-75.

11. Gow P, Testro AG, Hey P, Sinclair M. Letter to the editor: moderate alcohol use in fatty liver disease: don't throw the cabernet out with the bathwater. Hepatology 2020;71:1887-1888.

12. Loomba R, Lutchman G, Kleiner DE, Ricks M, Feld JJ, Borg BB, et al. Clinical trial: pilot study of metformin for the treatment of nonalcoholic steatohepatitis. Aliment Pharmacol Ther 2009;29:172-182.

13. Shen $Z$, Li Y, Yu C, Shen Y, Xu L, Xu C, et al. A cohort study of the effect of alcohol consumption and obesity on serum liver enzyme levels. Eur J Gastroenterol Hepatol 2010;22:820-825.

14. Wong VW, Chu WC, Wong GL, Chan RS, Chim AM, Ong A, et al. Prevalence of non-alcoholic fatty liver disease and advanced fibrosis in Hong Kong Chinese: a population study using protonmagnetic resonance spectroscopy and transient elastography. Gut 2012;61:409-415.

15. Moriya A, Iwasaki Y, Ohguchi S, Kayashima E, Mitsumune T, Taniguchi $\mathrm{H}$, et al. Roles of alcohol consumption in fatty liver: a longitudinal study. J Hepatol 2015;62:921-927.

16. Dunn W, Xu R, Schwimmer JB. Modest wine drinking and decreased prevalence of suspected nonalcoholic fatty liver disease. Hepatology 2008:47:1947-1954.

17. Sookoian S, Castaño GO, Pirola CJ. Modest alcohol consumption decreases the risk of non-alcoholic fatty liver disease: a meta-analysis of 43175 individuals. Gut 2014;63:530-532.

18. Chang Y, Ryu S, Kim Y, Cho YK, Sung E, Kim HN, et al. Low levels of alcohol consumption, obesity, and development of fatty liver with and without evidence of advanced fibrosis. Hepatology 2020;71:861-873.

19. Ajmera V, Belt $P$, Wilson LA, Gill RM, Loomba R, Kleiner $D E$, et al. Among patients with nonalcoholic fatty liver disease, modest alcohol use is associated with less improvement in histologic steatosis and steatohepatitis. Clin Gastroenterol Hepatol 2018;16:1511-1520. e5.

20. Vilar-Gomez E, Calzadilla-Bertot L, Wai-Sun Wong V, Castellanos $M$, Aller-de la Fuente $R$, Metwally $M$, et al. Fibrosis severity as a determinant of cause-specific mortality in patients with advanced nonalcoholic fatty liver disease: a multi-national cohort study. Gastroenterology 2018;155:443-457.e17.

21. Hajifathalian K, Torabi Sagvand B, McCullough AJ. Effect of alcohol consumption on survival in nonalcoholic fatty liver disease: a national prospective cohort study. Hepatology 2019;70:511-521.

22. VanWagner LB, Ning H, Allen NB, Ajmera V, Lewis CE, Carr JJ, et al. Alcohol use and cardiovascular disease risk in patients with nonalcoholic fatty liver disease. Gastroenterology 2017;153:1260-1272. e3.

23. Wood AM, Kaptoge S, Butterworth AS, Willeit P, Warnakula S, Bolton $T$, et al. Risk thresholds for alcohol consumption: combined analysis of individual-participant data for 599912 current drinkers in 83 prospective studies. Lancet 2018;391:1513-1523.

24. Gao B, Ahmad MF, Nagy LE, Tsukamoto H. Inflammatory pathways in alcoholic steatohepatitis. J Hepatol 2019;70:249-259.

25. Kolaczkowska E, Kubes P. Neutrophil recruitment and function in health and inflammation. Nat Rev Immunol 2013;13:159-175. 
Seonghwan Hwang, et al. Obesity and binge drinking induce steatohepatitis

26. Dominguez M, Miquel R, Colmenero J, Moreno M, García-Pagán $J C$, Bosch J, et al. Hepatic expression of CXC chemokines predicts portal hypertension and survival in patients with alcoholic hepatitis. Gastroenterology 2009;136:1639-1650.

27. Nischalke HD, Berger C, Lutz $P$, Langhans B, Wolter F, Eisenhardt $M$, et al. Influence of the CXCL1 rs4074 A allele on alcohol induced cirrhosis and HCC in patients of European descent. PLoS One 2013;8:e80848.

28. Gross B, Pawlak M, Lefebvre P, Staels B. PPARs in obesity-induced T2DM, dyslipidaemia and NAFLD. Nat Rev Endocrinol 2017;13:3649.

29. Xu MJ, Cai Y, Wang H, Altamirano J, Chang B, Bertola A, et al. Fatspecific protein 27/CIDEC promotes development of alcoholic steatohepatitis in mice and humans. Gastroenterology 2015;149:10301041.e6.

30. Zhang W, Sun Q, Zhong W, Sun X, Zhou Z. Hepatic peroxisome proliferator-activated receptor gamma signaling contributes to alcoholinduced hepatic steatosis and inflammation in mice. Alcohol Clin Exp Res 2016;40:988-999.

31. Torres-Hernandez A, Wang W, Nikiforov Y, Tejada K, Torres L, Kalabin $A$, et al. $\gamma \delta T$ cells promote steatohepatitis by orchestrating innate and adaptive immune programming. Hepatology 2020;71:477494.

32. Wang X, Gao B. үסT Cells and CD1d, novel immune players in alcoholic and nonalcoholic steatohepatitis? Hepatology 2020;71:408410.

33. Xu D, Han H, He Y, Lee H, Wu D, Liu F, et al. A hepatocyte-mimicking antidote for alcohol intoxication. Adv Mater 2018;30:e1707443.

34. Jaeschke H. Neutrophil-mediated tissue injury in alcoholic hepatitis. Alcohol 2002;27:23-27.

35. Bertola A, Park 0, Gao B. Chronic plus binge ethanol feeding synergistically induces neutrophil infiltration and liver injury in mice: a critical role for E-selectin. Hepatology 2013;58:1814-1823.

36. Koyama Y, Brenner DA. Liver inflammation and fibrosis. J Clin Invest 2017:127:55-64.

37. Bataller R, Gao B. Liver fibrosis in alcoholic liver disease. Semin Liver Dis 2015;35:146-156

38. Bedard K, Krause KH. The NOX family of ROS-generating NADPH oxidases: physiology and pathophysiology. Physiol Rev 2007;87:245313.

39. Lopez AF, Williamson DJ, Gamble JR, Begley CG, Harlan JM, Klebanoff $\mathrm{SJ}$, et al. Recombinant human granulocyte-macrophage colony-stimulating factor stimulates in vitro mature human neutrophil and eosinophil function, surface receptor expression, and survival. J Clin Invest 1986;78:1220-1228.

40. Girard D, Paquet ME, Paquin R, Beaulieu AD. Differential effects of interleukin-15 (IL-15) and IL-2 on human neutrophils: modulation of phagocytosis, cytoskeleton rearrangement, gene expression, and apoptosis by IL-15. Blood 1996;88:3176-3184.

41. Matsumoto M, Hada N, Sakamaki Y, Uno A, Shiga T, Tanaka C, et al. An improved mouse model that rapidly develops fibrosis in nonalcoholic steatohepatitis. Int J Exp Pathol 2013;94:93-103.

42. Hwang $S$, Gao B. How does your fat affect your liver when you drink? J Clin Invest 2019;129:2181-2183.

43. Sebastian BM, Roychowdhury S, Tang $H$, Hillian AD, Feldstein AE, Stahl GL, et al. Identification of a cytochrome P4502E1/Bid/C1q-dependent axis mediating inflammation in adipose tissue after chronic ethanol feeding to mice. J Biol Chem 2011;286:35989-35997.

44. Kim SJ, Feng D, Guillot A, Dai S, Liu F, Hwang S, et al. Adipocyte death preferentially induces liver injury and inflammation through the activation of chemokine (C-C Motif) receptor 2-positive macrophages and lipolysis. Hepatology 2019;69:1965-1982.

45. Rinella ME, Tacke F, Sanyal AJ, Anstee QM; participants of the AASLD/EASL Workshop. Report on the AASLD/EASL Joint Workshop on clinical trial endpoints in NAFLD. Hepatology 2019;70:1424-1436.

46. Sanyal AJ. Past, present and future perspectives in nonalcoholic fatty liver disease. Nat Rev Gastroenterol Hepatol 2019;16:377-386.

47. Tilg $H$, Moschen AR. Evolution of inflammation in nonalcoholic fatty liver disease: the multiple parallel hits hypothesis. Hepatology 2010;52:1836-1846.

48. Gao B, Tsukamoto H. Inflammation in alcoholic and nonalcoholic fatty liver disease: friend or foe? Gastroenterology 2016;150:17041709.

49. Bertola A, Bonnafous S, Anty R, Patouraux S, Saint-Paul MC, lannelli $A$, et al. Hepatic expression patterns of inflammatory and immune response genes associated with obesity and NASH in morbidly obese patients. PLoS One 2010;5:e13577.

50. Hwang S, He Y, Xiang X, Seo W, Kim SJ, Ma J, et al. Interleukin-22 ameliorates neutrophil-driven nonalcoholic steatohepatitis through multiple targets. Hepatology 2020;72:412-429.

51. Hwang $S$, Wang $X$, Rodrigues RM, Ma J, He Y, Seo W, et al. Protective and detrimental roles of p38a MAPK in different stages of nonalcoholic fatty liver disease. Hepatology. 2020 May 28. doi: 10.1002/hep.31390.

52. Ji C. New Insights into the pathogenesis of alcohol-induced ER stress and liver diseases. Int J Hepatol 2014;2014:513787.

53. Wang S, Pacher P, De Lisle RC, Huang H, Ding WX. A mechanistic review of cell death in alcohol-induced liver injury. Alcohol Clin Exp Res 2016;40:1215-1223.

54. Farrell G, Schattenberg JM, Leclercq I, Yeh MM, Goldin R, Teoh N, et al. Mouse models of nonalcoholic steatohepatitis: toward optimization of their relevance to human nonalcoholic steatohepatitis. Hepatology 2019;69:2241-2257.

55. Tsuchida T, Lee YA, Fujiwara N, Ybanez M, Allen B, Martins S, et al. A simple diet- and chemical-induced murine NASH model with rapid progression of steatohepatitis, fibrosis and liver cancer. J Hepatol 


\section{CLINICAL and MOLECULAR}

2018:69:385-395.

56. von Vietinghoff S, Ley K. Homeostatic regulation of blood neutrophil counts. J Immunol 2008;181:5183-5188.

57. Sinclair J, Lambrecht L, Smith EL. Hepatic alcohol dehydrogenase activity in chick hepatocytes towards the major alcohols present in commercial alcoholic beverages: comparison with activities in rat and human liver. Comp Biochem Physiol B 1990;96:677-682.

58. Hart CL, Morrison DS, Batty GD, Mitchell RJ, Davey Smith G. Effect of body mass index and alcohol consumption on liver disease: analysis of data from two prospective cohort studies. BMJ 2010;340:c1240.

59. Everitt H, Hu M, Ajmo JM, Rogers CQ, Liang X, Zhang R, et al. Ethanol administration exacerbates the abnormalities in hepatic lipid oxidation in genetically obese mice. Am J Physiol Gastrointest Liver Physiol 2013;304:G38-G47.

60. Minato T, Tsutsumi M, Tsuchishima M, Hayashi N, Saito T, Matsue $Y$, et al. Binge alcohol consumption aggravates oxidative stress and promotes pathogenesis of NASH from obesity-induced simple steatosis. Mol Med 2014;20:490-502.

61. Alwahsh SM, Xu M, Schultze FC, Wilting J, Mihm S, Raddatz D, et al. Combination of alcohol and fructose exacerbates metabolic imbalance in terms of hepatic damage, dyslipidemia, and insulin resistance in rats. PLoS One 2014;9:e104220.

62. Duly AM, Alani B, Huang EY, Yee C, Haber PS, McLennan SV, et al. Effect of multiple binge alcohol on diet-induced liver injury in a mouse model of obesity. Nutr Diabetes 2015;5:e154.

63. Song M, Chen T, Prough RA, Cave MC, McClain CJ. Chronic alcohol consumption causes liver injury in high-fructose-fed male mice through enhanced hepatic inflammatory response. Alcohol Clin Exp Res 2016;40:518-528.

64. Warner DR, Liu H, Miller ME, Ramsden CE, Gao B, Feldstein AE, et al. Dietary linoleic acid and its oxidized metabolites exacerbate liver injury caused by ethanol via induction of hepatic proinflammatory response in mice. Am J Pathol 2017;187:2232-2245. 